\section{Ribosomal 5S RNA}

\section{from a Correspondent in Biochemical Genetics}

Two years ago Holley and his collaborators determined the base sequence of yeast alanine transfer RNA, the first RNA molecule to be sequenced. Subsequently Zachau and co-workers reported the sequences of two yeast serine transfer RNAs, Madison and co-workers that of yeast tyrosine transfer RNA and Khorana's group that of the yeast phenylalanine transfer RNA. In these cases the major problem lay in the isolation of several hundred milligrams of pure transfer RNA to sequence. The actual sequence determination was facilitated by the presence of unusual bases in these molecules, thereby helping to obtain overlaps of the fragments produced by digestion of the RNA with either pancreatic or $\mathrm{Tl}$ ribonuclease.

In this issue of Nature (page 735) Brownlee, Sanger and Barrell report the sequence of the $E$. coli $5 S$ ribosomal RNA. This RNA occurs in the $50 \mathrm{~S}$ ribosomal sub-unit and may be purified much more easily than a transfer RNA molecule. The techniques they used, which they themselves had developed, depend on handling very small amounts (about 100 micrograms) of RNA highly labelled with ${ }^{32} \mathrm{P}$. The difficulties in solving this sequence lay in its length of 120 nucleotides and in the absence of any unusual bases. To establish the sequence unambiguously therefore roquired the fractionation and sequence determination. of many partial digestion products. The molecule, when folded up to allow some base pairing, looks quite different from a transfer RNA molecule. Of particular interest is the apparent homology between the two halves of the molecule. The authors suggest that this may be of evolutionary significance, though it is possibly more interesting to speculate that this homology reflects some symmetry in the construction of the $50 S$ ribosomal sub-unit. This work represents a significant advance in the methodology of sequencing large RNA molecules.

\section{Feline Vision}

\section{from a Correspondent in Neurophysiology}

THE receptive field of a unit in an animal's visual system has come to be known as that area of retina over which light stimulation causes a change in excitability of the neurone, usually measured as a change in the rate of production of action potentials. Since Hartline's classic work on the frog optic nerve, in which he discovered single fibres carrying responses to the "on" and "off" of a light stimulus, as well as fibres responding to both, a vast amount of information about the response properties of visual units has been amassed. Hubel and Wiesel, in particular ( $J$. Neurophysiol., 28, 229; 1965), have investigated receptive field organization of units at several levels in the cat visual system, and have apparently demonstrated the existence of a hierarchy in which increasingly complex features of a visual stimulus are abstracted. There is some evidence that such a hierarchical organization also exists in the human visual system, and artificial pattern recognition devices have been designed to perform feature detection on a hierarchical basis.

Rodieck has recently found a new type of receptive ficld for ganglion cells in the cat's retina (Science,
157, $90:$ 1967). In general these have a centresurround organization, in which the properties of the centre oppose those of the surround: if the centre responds to the on of a stimulus, then the surround responds to its off, and vice-versa. In two cats, however, one decerebrate and the other anaesthetized with nitrous oxide, Rodieck found two ganglion cells which responded to contrast anywhere within their receptive fields. These were between $1.5^{\circ}$ and $2 \cdot 5^{\circ}$ in diameter (in terms of retinal angle) and possessed several unusual features. A black or white disk on a grey background, presented to the centre of the receptive field of either unit, caused an inhibition of firing: its removal was followed by reversion to the previous, maintained, firing rate, with no added "off" response. More complex stimuli, such as chessboard patterns, had similar effects: it seemed that contrast, and only contrast, was causing changes in firing rate. Movement of the stimulus at speeds of up to $10 \%$ sec and rotations of the chessboard pattern had no effect, and it did not matter whether the stimulus was brighter or dimmer than the surround.

This discovery again poses the question of how much peripheral data processing is done in the mammalian visual system. Barlow and Hill have already shown that in the rabbit retina there are units at the ganglion cell level - and possibly as far out as the bipolar colls--which can detect movement of objects in the visual field in particular directions. Maturana, Lettvin, McCullough and Pitts have suggested that the frog's retina extracts four classes of feature from visual input, sending information to the optic tectum about small round objects ("bugs"), moving edges, contrast and "dimming" of stimuli. This peripheral organization of input is in marked contrast to the hierarchical organization described by Hubel and Wiesel for the cat, so the recent findings of Rodieck and also of Stone and Fabian (Science, 152, 1277; 1966) serve to complicate the issue as they are not yet explained by the known details of retinal organization. Dowling and Boycott, working on the Primate retina (Proc. Roy. Soc., B, 166, 80-111: 1966), found quite complicated patterns of synaptic organization which could well underlie similar functions in man and the rhesus monkey. They describe synapses involving, in the inner plexiform layer, bipolar, amacrine and ganglion cell processes. There seems to be ample opportunity for the lateral spread of excitation and inhibition necessary for movement detection, as well as in border, or contrast, enhancement. There is also the possibility of similar synaptic organization in the outer plexiform layer where horizontal cells might also be involved in the lateral spread of information between receptors.

\section{Nucleoli are Temperature Sensitive}

\section{from a Correspondent in Cell Biology}

Simard and. Bernhard (J. Cell Biol., 34, 61; 1967) have discovered that exposure of cultured mammalian. cells to temperatures above normal selectively and reversibly affects the structure and function of nucleoli. In 1965 Gharpure reasoned that since DNA viruses fail to replicate in cells given a temperature shock (15 min at $45^{\circ} \mathrm{C}$ ) before infection, while RNA viruses replicate normally, there is some temperature sensitive step in DNA dependent RNA synthesis. Simard and 